\title{
Automatic CFAR Ship Detection in Single-Channel Range-Compressed Airborne Radar Data
}

\author{
Sushil Kumar Joshi, Stefan V. Baumgartner \\ Microwaves and Radar Institute, German Aerospace Center (DLR), Wessling, Germany \\ Email: Sushil.Joshi@dlr.de
}

\begin{abstract}
In the paper a novel ship detection method using single-channel rangecompressed airborne radar data is proposed. The target detection threshold is computed in range-Doppler domain using constant false alarm rate (CFAR) based sea clutter models. Appropriate training data for computing a bias-free threshold is automatically extracted after performing two major steps. The first step is target pre-detection and cancellation in time domain with the proposed pre-detection algorithm. The second step is clutter normalization using the remaining data in Doppler domain. Suitable sea clutter models are then fit to determine the threshold. Real single-channel range-compressed data from DLR's F-SAR system is used to verify the proposed method.
\end{abstract}

\section{Introduction}

Ship detection and monitoring is an important application for ensuring maritime security. Ground based systems for ship detection have limited range and many ships, especially the smaller ones, are not equipped with Automatic Identification System (AIS) transreceivers for transmitting their geographical positions. Remotely sensed images acquired from either spaceborne or airborne platforms over the sea surface are considered to be very efficient for continuous monitoring. Especially synthetic aperture radar (SAR) images are of high relevance in such applications due to their all-weather independent and day night acquisition capabilities.

Ship detection using SAR is a well-known topic with many publications available in the literature [1], [2]. One of the popular detection methods is based on the constant false alarm rate (CFAR) [3]. CFAR based sea clutter models were found to fit well for the target detection in fully focused SAR images, especially at shallow incidence angles. However, there exists limited work for evaluating and applying these models on range-compressed (RC) radar data. Using RC data is very attractive, especially under the viewpoint of real time ship detection, since no comprehensive and time consuming SAR processing has to be carried out. By transferring the $\mathrm{RC}$ data to range-Doppler domain, the probability of detection can be increased, especially for the ships moving fast enough so that their signals are shifted into the exo-Clutter region [4], [5].

The present paper proposes a novel method of ship detection using RC data, suitable for real time applications. The target detection is carried out in range-Doppler domain. CFAR based sea clutter models are implemented to determine the detection threshold. The accuracy of such a threshold however relies on the proper selection of the training data. Suitable training data without disturbing ship signals, which describe the background ocean statistics correctly, is needed for computing a valid CFAR threshold. Therefore this paper also proposes a strategy to automatically extract suitable training data. The proposed methodology is tested on real single-channel RC radar data acquired with DLR's F-SAR airborne sensor.

The remainder of this paper is organized as follows: The basic idea of the overall proposed method is provided in section 2 and the training data selection method is presented in section 3. A brief description of the sea clutter models and the detection results are provided in section 4 followed by the final conclusion. 


\section{Basic idea}

Ships are detected in the range-Doppler domain. If the ships, even with low radar cross section (RCS), move with a certain line-of-sight (LOS) velocity, they will be shifted to the exo-clutter region. Detection is then possible if this shift is larger than half the clutter bandwidth.

The basic idea of the proposed ship detection method using single-channel RC radar data is outlined as follows:

1. Extraction of a data block from the RC radar data in time domain. The data block in our case for the F-SAR system has 512 range and 128 azimuth samples. The selected number of azimuth samples is typically called coherent processing interval (CPI).

2. Transformation of each data block to range-Doppler domain via azimuth fast Fourier transform (FFT).

3. Estimation of the average Doppler spectrum.

4. Normalization over Doppler to get a "flat spectrum".

5. Estimation of the sea clutter statistics.

6. Computation of the CFAR threshold.

Steps 2-6 are repeated for each data block, first along the range and then along the azimuth direction. For each data block CFAR threshold is computed. A valid threshold can only be obtained if "ocean only" training data is used for the sea clutter modeling. The presence of bright targets and other high clutter peaks in the region of interest contaminates the training data. Such contamination drastically degrades the detection performance. Therefore, predetection and cancellation of such interfering target signals is imperative prior to step 3 .

\section{Training data selection}

With a proper selection of ocean training data the sea clutter statistics can be estimated accurately. An example of the presence of a ship signal in the data in time domain is shown in Figure 1.

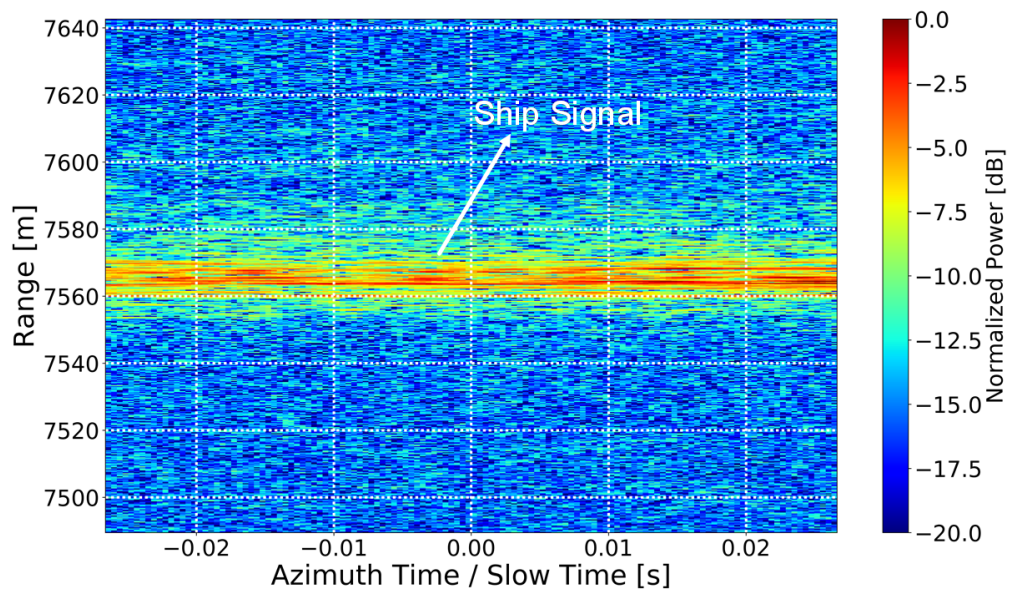

Figure 1. Bright ship signal present in a F-SAR X-band HH polarized data block. The image has 512 range samples and 128 azimuth samples. For visualization purposes, the data is normalized to the maximum power.

As shown in Figure 1, ships often appear as brigh pixels compared to its background, called sea clutter. Therefore, detecting and cancelling of such high peaks is needed for obtaining "ocean only" training data. Several methods for excluding bright target signals, for instance 
based on truncated statistics or iterative censoring are proposed in the literature [6]. These methods can be used to exclude the potential target peaks but they are computationally inefficient. We propose a rather simple and effective method of target pre-detection with real time capability.

A RC data patch containing a certain number of azimuth samples and the entire range is first extracted. An incoherent summation over azimuth is performed and a pre-detection threshold is computed. The computed threshold is adaptive along range because the received radar backscatter is range and incidence angle dependent as shown in Figure 2.

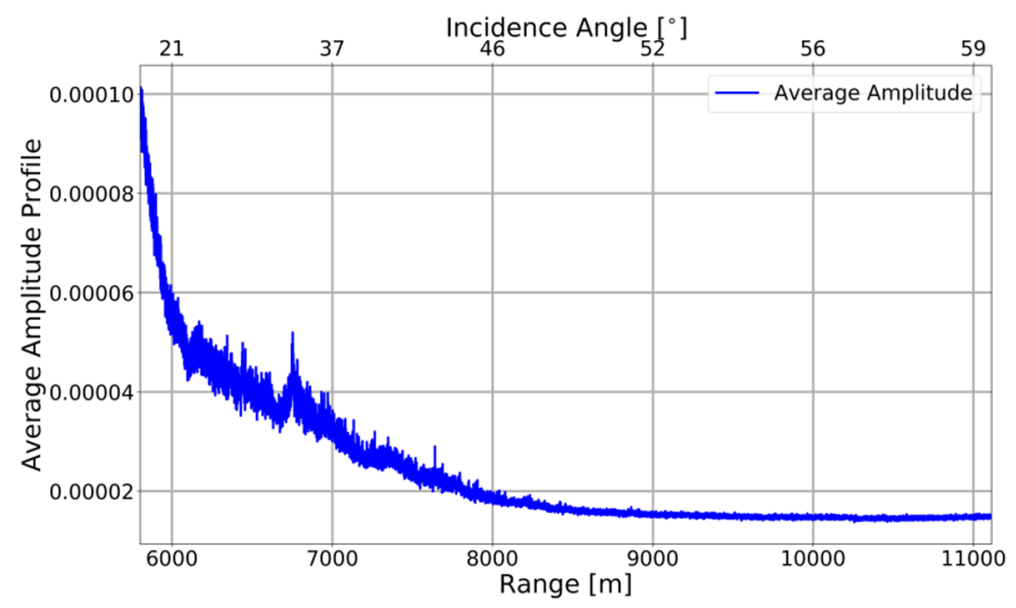

Figure 2. Average amplitude profile of a HH polarized RC F-SAR X-band data batch.

If $r$ is the range and $A(r)$ is the average ampitude profile (cf. Figure 2), then the pre-detection threshold $\eta_{\text {pre }}(r)$ is given as

$$
\eta_{p r e}(r)=\tilde{A}(r)+f \cdot \operatorname{SG}(\sigma(r))
$$

where $\tilde{A}(r)$ is the median of $A(r), f>1$ and SG is the Savitky Golay filter. The standard deviation $\sigma(r)$ in (1) is computed as

$$
\sigma(r)=k \cdot M A D(r)
$$

where $k$ is a scale factor with $k \approx 1.4826$ representing the 0.75 quantile of the standard Gaussian distribution, and $M A D(r)$ is the median absolute deviation which is written as

$$
M A D(r)=\operatorname{median}(|A(r)-\tilde{A}(r)|) .
$$

To illustrate this, the computed adaptive pre-detection threshold and the averave amplitude over range profile of a RC X-band F-SAR data block is shown in Figure 3. 


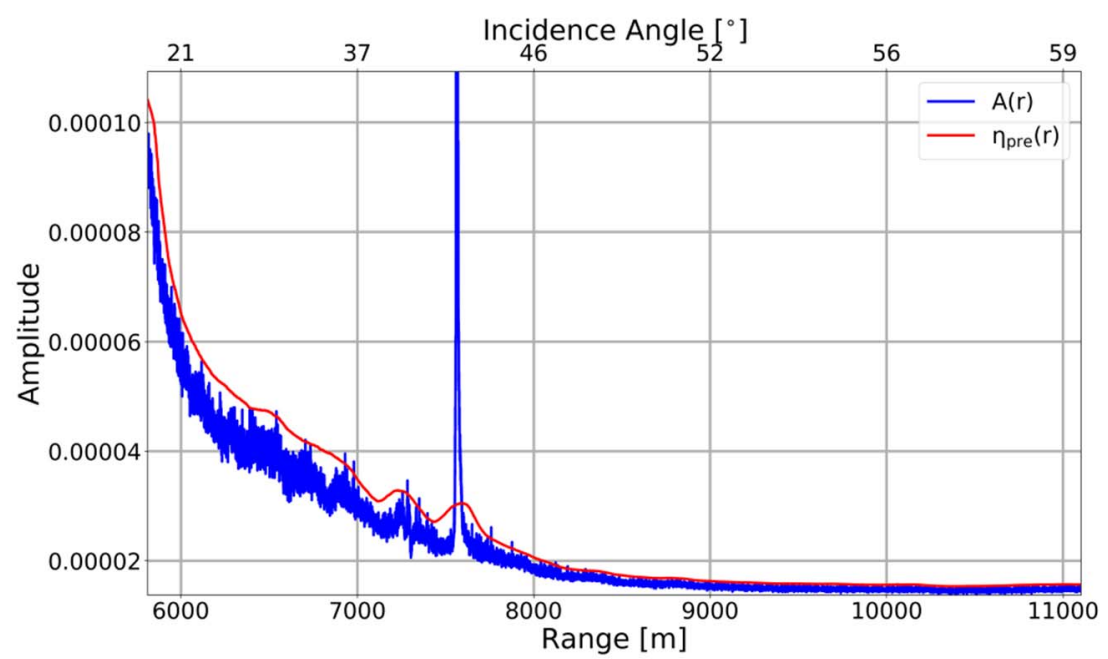

Figure 3. Average amplitude range profile $A(r)$ (blue) with range dependent pre-detection threshold $\eta_{\text {pre }}(r)$ (red) for a RC X-band F-SAR data. A high target peak (= ship) is present at a range of approximately $7500 \mathrm{~m}$.

For computing this threshold, the window size of the moving median filter was chosen as 625 , which is large enough to cover the maximum expected ship length in range direction. Furthermore, a polynomial order of 2 and a window size of 625 for the SG filter and $f=4$ (cf. (2)) were chosen. For the X-band F-SAR data acquisition geometry, these values were found suitable to successfully detect the outliers.

The proposed pre-detection algorithm is applied to a real X-band $\mathrm{HH}$ polarized RC data in time domain. The results are shown in Figure 4.
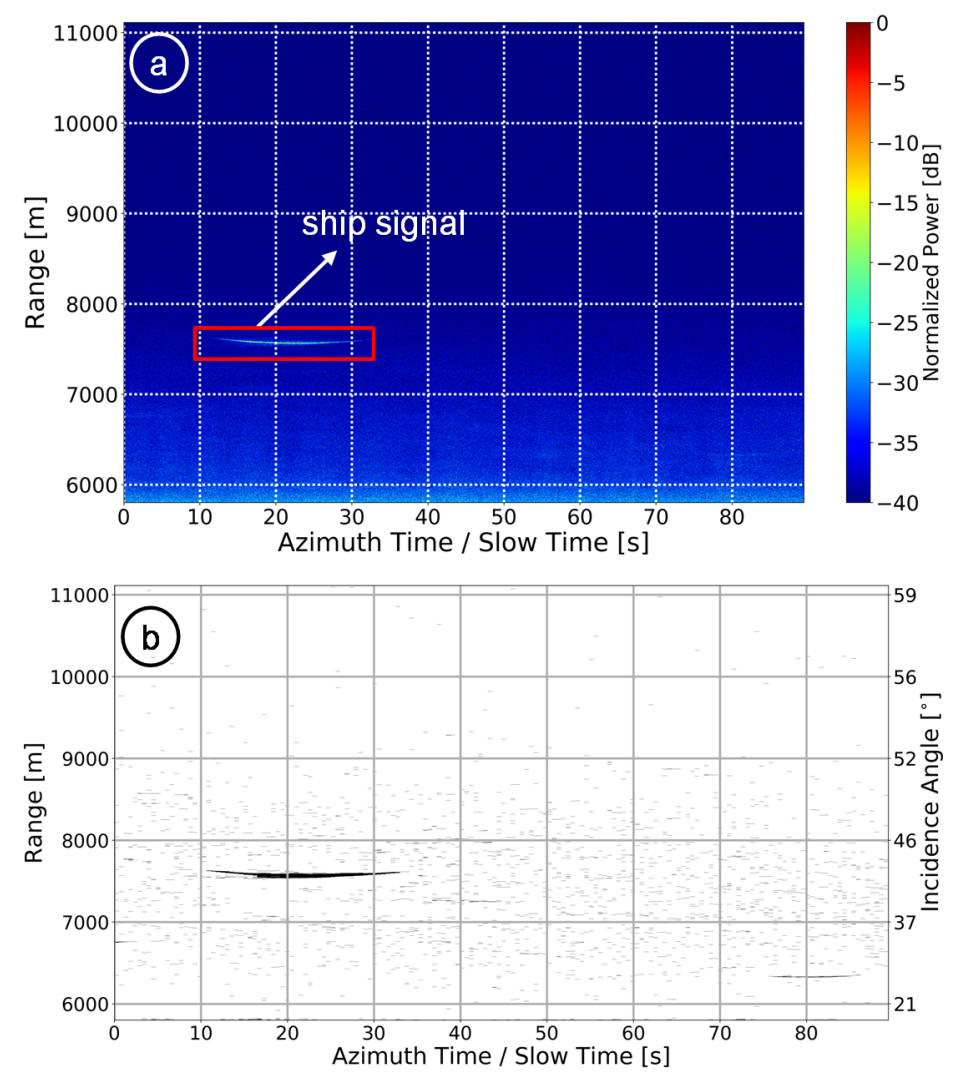

Figure 4. (a) Real single-channel RC X-band F-SAR radar data. The ship signal is highlighted in the figure. (b) Binary pre-detection map after applying the proposed pre-detection algorithm .The pre-detected ship (left) and spiky clutter can be clearly seen in (b). 
After cancelling the target's azimuth lines, the remaining "training data" is transformed to range-Doppler domain. The data in this domain vary along the Doppler frequency. Therefore, the steps of single CFAR threshold computation in time domain cannot be applied one to one in Doppler domain. The idea is then to normalize the clutter and noise power to $0 \mathrm{~dB}$ so that a single and Doppler frequency independent CFAR threshold can be computed [7], [8]. This is illustrated in Figure 5.
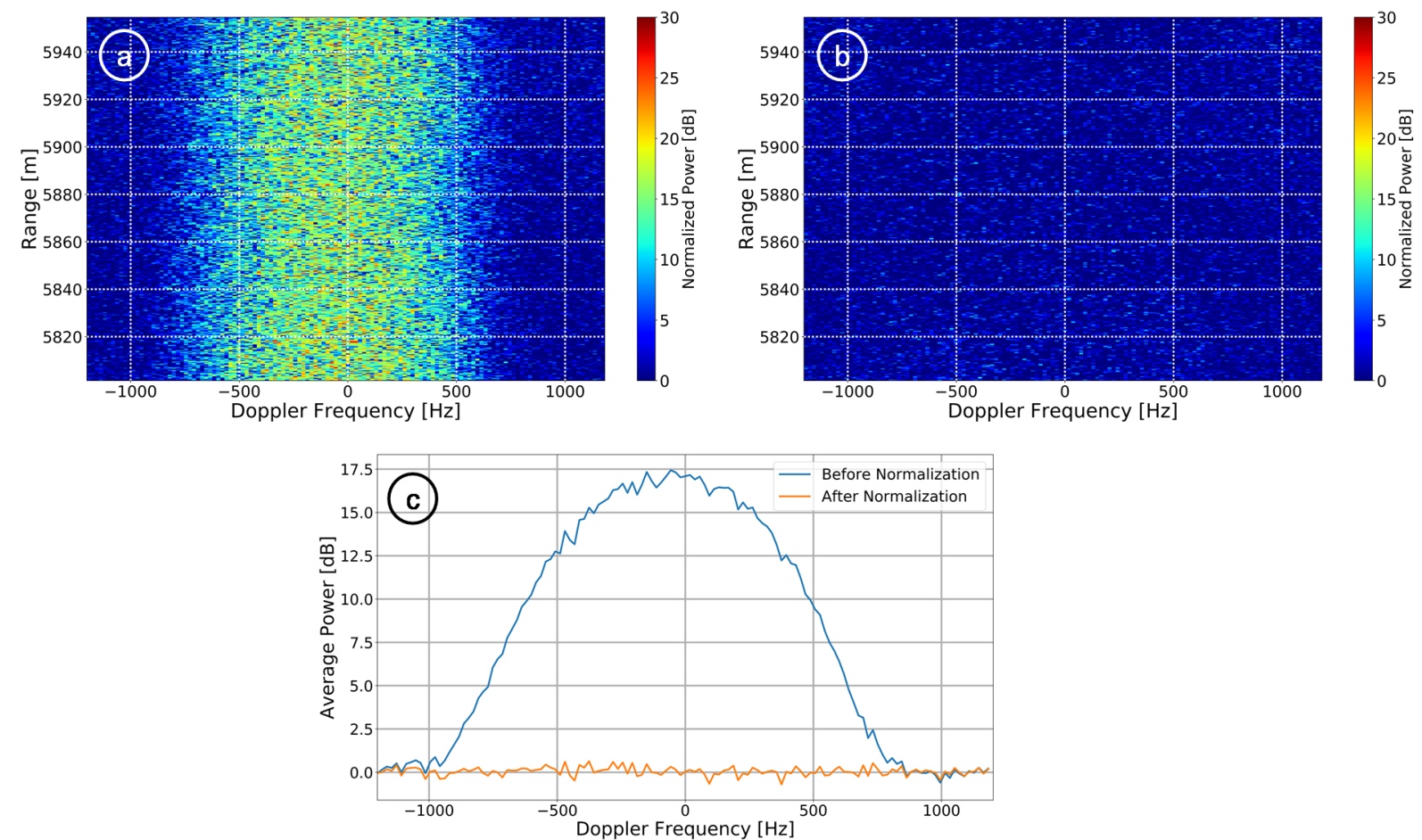

Figure 5. (a) Range-Doppler image, (b) normalization of the range-Doppler image, (c) average Doppler spectrum before and after normalization. Note that the bright peaks are already cancelled in (a) before spectrum estimation and normalization. The images in (a) and (b) were generated using 512 range and 128 azimuth samples.

For performing the normalization over Doppler, an average Doppler spectrum (blue curve in Figure 5(c)) is first estimated. The estimated Doppler spectrum is then used to whiten the data shown in Figure 5(a) to get as a result the normalized data shown in Figure 5(b). The normalized data is used as training data for computing the sea clutter statistics.

\section{Model fitting and threshold estimation}

The K-distribution is one of the widely used sea clutter models [9]. Comprehensive representation of the Gamma distributed texture and the Gaussian distributed homogeneous clutter makes this distribution model to work very well for a high resolution homogeneous clutter environment. An example of the ship detection for a desired false alarm rate of $10^{-6}$ by assuming a K-distribution is shown in Figure 6. The CFAR threshold is computed in rangeDoppler domain for each data block separately (cf. section 2). For visualization purposes, the detections obtained in range-Doppler domain are conterted to time domain. 


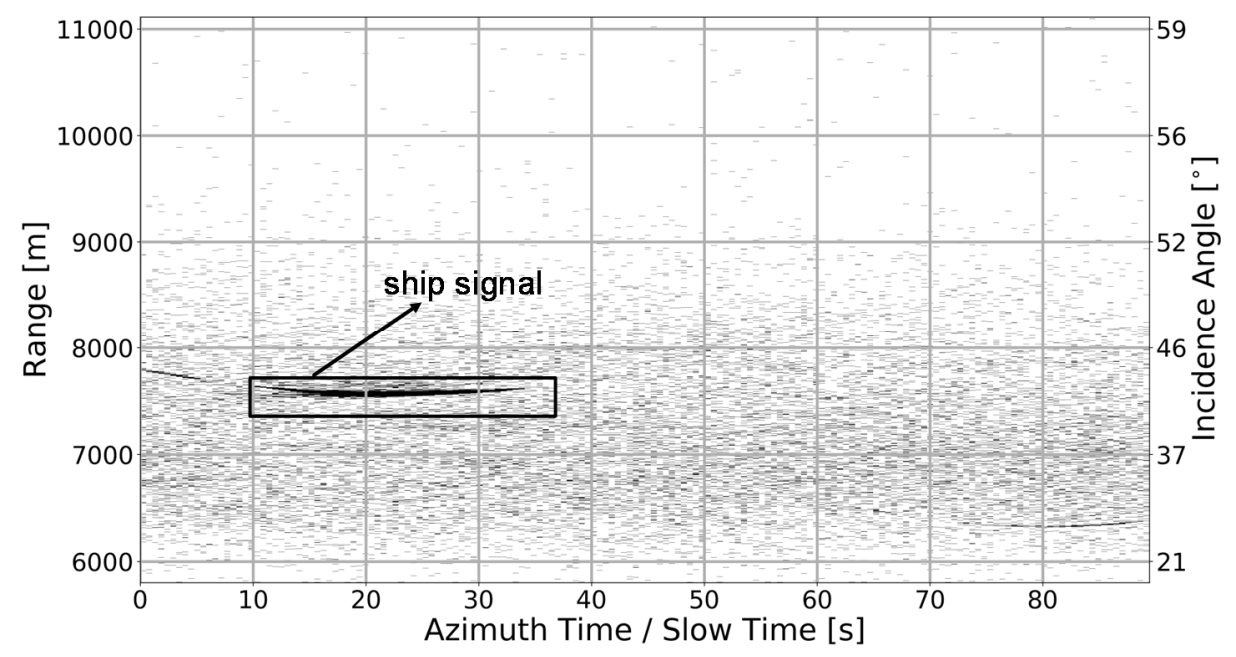

Figure 6. Binary detection map obtained after applying the K-distribution based CFAR ship detection threshold. The detection map corresponds to the data shown in Figure 4. The ship signal and spiky clutter are clearly visible.

It can be seen in Figure 6 that high intensity discrete sea spikes are present in the near and mid ranges of the data $\left(\approx 21\right.$ to $\approx 50^{\circ}$ incidence angle). These spikes lead to extremely high false alarms [10].

Rather than the K-distribution the K-Rayleigh distribution is able to model these spikes by adding an extra Rayleigh component. The K-Rayleigh distribution works well in the near and mid ranges but found unsuitable for the far range due to the low clutter-to-noise ratio (CNR) [11]. Therefore, in this paper a clutter model known as tri-modal discrete texture (3MD) model is used for the far range [6].

The performance of these models are evaluated in terms of the false alarm rate errors. They are computed for the near range $\left(15^{\circ}-30^{\circ}\right.$ incidence angle), mid range $\left(30^{\circ}-50^{\circ}\right)$ and far range $\left(>50^{\circ}\right)$ of the data shown in Figure 4 . The error is the ratio between the estimated (actual) false alarm rate and the set false alarm rate of $10^{-6}$. Before carrying out the evaluation bright targets are removed using the pre-detection algorithm. In the optimum case, if there are no targets and no spiky clutter, the ratio is one. The obtained results are shown in Table 1.

Table 1. False alarm rate errors at different ranges for a set false alarm rate of $\mathbf{1 0}^{-\mathbf{6}}$.

\begin{tabular}{|l|l|l|l|}
\hline Slant range $[\mathrm{m}]$ & K-distribution & K-Rayleigh distribution & 3MD model \\
\hline Near range & 80.5 & $\mathbf{1 . 3 1}$ & 149.2 \\
\hline Mid range & 112.1 & $\mathbf{1 . 6 8}$ & 135.9 \\
\hline Far range & 3.08 & - & $\mathbf{1 . 5 6}$ \\
\hline
\end{tabular}

From Table 1, it can be observed that the false alarm rate error is maximum in case of the Kdistribution and the 3MD model in the near and mid range, but the ratio is close to one for the far range of the data. The K-Rayleigh distribution yields the least error in the near and mid range, but is not suited in the far range due to low CNR. 
Based on these analyses, the chosen model for the ship detection in RC F-SAR X-band data are the K-Rayleigh distribution for the near and mid ranges and the 3MD model for the far range. The detection results are shown in Figure 7 for the data shown in Figure 4.

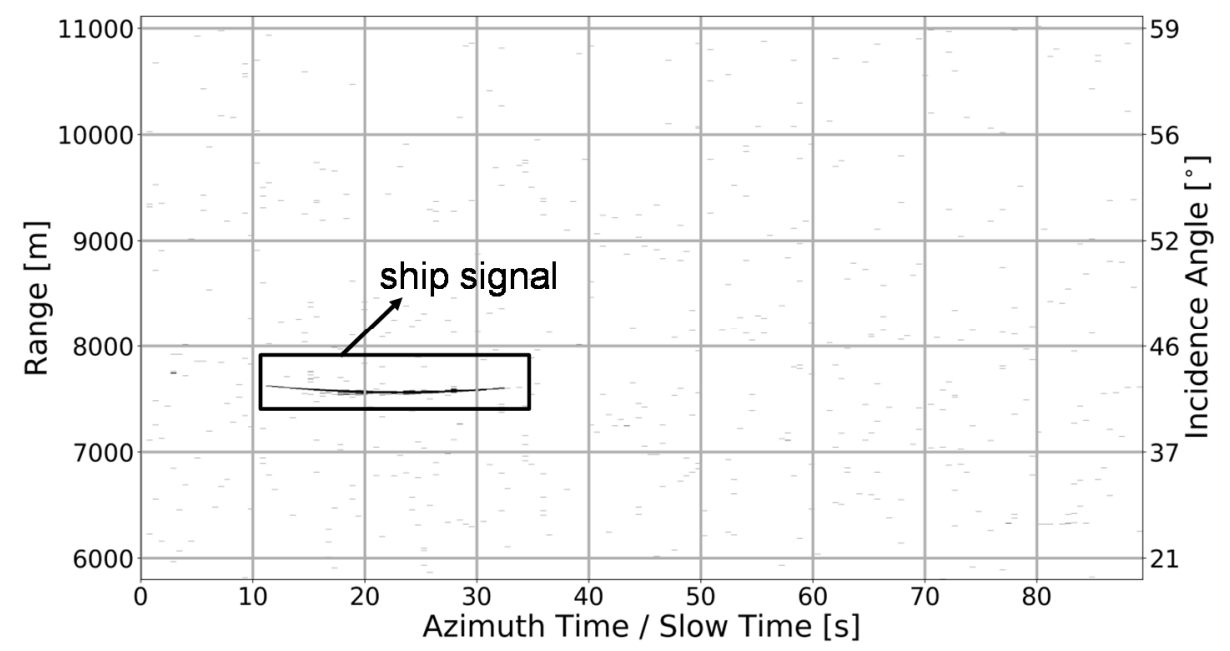

Figure 7. Binary detection map after applyint the K-Rayleigh distribution based CFAR threshold in near and mid range and the $3 \mathrm{MD}$ model based CFAR threshold in far range. The desired false alarm rate was set to $\mathbf{1 0}^{-6}$.

As observed in Figure 7 the spiky clutter in the near and mid ranges are not detected after using the K-Rayleigh distribution function for that ranges (compare to Figure 6).

\section{Conclusion}

A novel ship detection approach using single-channel range-compressed (RC) data was proposed in the paper. The proposed target pre-detection algorithm in time domain followed by the clutter normalization in Doppler domain generate appropriate training data for computing a valid and accurate CFAR detection threshold. The conventional K-distribution function leads to high false alarms in the near and mid range of the data. We found that, at least for the available RC X-band F-SAR radar data, as sea clutter models the K-Rayleigh distribution for the near and mid range and the 3MD model for the far range lead to the best results. However, further investigations using a larger amount of F-SAR data acquired during different sea states are recommended.

\section{References}

[1] K. Eldhuset, "An automatic ship and ship wake detection system for spaceborne sar images in coastal regions," IEEE Trans. Geosci. Remote Sens., vol. 34, no. 4, pp. 1010-1019, 1996.

[2] J.-C. Souyris, C. Henry, and F. Adragna, "On the use of complex SAR image spectral analysis for target detection: assessment of polarimetry," Geosci. Remote Sensing, IEEE Trans., vol. 41, no. 12, pp. 2725-2734, 2003.

[3] S. Brusch, S. Lehner, T. Fritz, M. Soccorsi, A. Soloviev, and B. Van Schie, "Ship surveillance with TerraSAR-X," IEEE Trans. Geosci. Remote Sens., vol. 49, no. 3, pp. 1092-1103, 2011.

[4] S. K. Joshi, S. V Baumgartner, and G. Aerospace, "Sea clutter model comparison for ship detection using single channel airborne raw SAR data Sea clutter modelling," EUSAR 2018 12th Eur. Conf. Synth. Aperture Radar, Proc., no. 1, pp. 731-735, 2018.

[5] S. V Baumgartner, "Linear and Circular ISAR Imaging of Ships Using DLR' $s$ Airborne Sensor F-SAR,” in IET Radar, 2017. 
[6] C. H. Gierull and I. Sikaneta, "A Compound-Plus-Noise Model for Improved Vessel Detection in Non-Gaussian SAR Imagery," IEEE Trans. Geosci. Remote Sens., vol. 56, no. 3, pp. 1444-1453, 2018.

[7] L. Rosenberg and S. Watts, "Model based coherent detection in medium grazing angle sea-clutter," in 2016 IEEE Radar Conference, RadarConf 2016, 2016.

[8] S. Watts and L. Rosenberg, "A comparison of coherent and non-coherent radar detection performance in radar sea clutter," in International Conference on Radar Systems (Radar 2017), 2017, pp. 1-6.

[9] K. D. Ward, "Compound representation of high resolution sea clutter," Electron. Lett., vol. 17 , no. 16 , pp. 561-563, 1981.

[10] D. J. Crisp, L. Rosenberg, N. J. Stacy, and D. Yunhan, "Modelling X-band sea clutter with the K-distribution: Shape parameter variation," Int. Radar Conf. - Surveill. a Safer World (RADAR '09), pp. 1-6, 2009.

[11] L. Rosenberg, S. Watts, and S. Bocquet, "Application of the K+Rayleigh distribution to high grazing angle sea-clutter," in 2014 International Radar Conference, Radar 2014, 2014. 